\title{
Electrochemical sensor based on poly (aspartic acid) modified carbon paste electrode for paracetamol determination
}

\author{
Mohammed Monirul Islam ${ }^{1, *}$, MD Arifuzzaman ${ }^{2}$, Sayeed Rushd ${ }^{3}$, MD Kamrul Islam ${ }^{4}$, and Muhammad \\ Muhitur Rahman ${ }^{5}$ \\ ${ }^{1}$ Department of Biomedical Sciences, College of Clinical Pharmacy, King Faisal University, \\ Al-Ahsa, 31982, Saudi Arabia \\ ${ }^{2}$ Department of Civil and Environmental Engineering, College of Engineering, King Faisal University, \\ Al-Ahsa, 31982, Saudi Arabia \\ ${ }^{3}$ Department of Chemical Engineering, College of Engineering, King Faisal University, Al-Ahsa, \\ 31982, Saudi Arabia \\ ${ }^{4}$ Department of Civil and Environmental Engineering, College of Engineering, King Faisal University, \\ Al-Ahsa, 31982, Saudi Arabia \\ ${ }^{5}$ Department of Civil and Environmental Engineering, College of Engineering, King Faisal University, \\ Al-Ahsa, 31982, Saudi Arabia \\ *E-mail: mislam@kfu.edu.sa
}

Received: 3 November 2021 / Accepted: 15 December 2021 / Published: 5 January 2022

Electrocatalytic determination of paracetamol (PRT) in presence of cetirizine $(\mathrm{CN})$ at the carbon paste sensor (CPS) modified with poly aspartic acid (PA) via electrochemical polymerization method was discussed. Oxidation of PRT at the poly (aspartic acid) modified carbon paste sensor (PAMCPS) followed at low overpotential and voltammetric information showed that it was an absolutely surface controlled response involving equal number of electrons and protons transfer. The modified sensor displays great catalytic activity for the oxidation of PRT with great sensitivity over the concentration range of $10.0 \mu \mathrm{M}$ to $105.0 \mu \mathrm{M}$, with the detection limit (DL) of $53.37 \mathrm{nM}(\mathrm{S} / \mathrm{N}=3)$ and quantification limit $(\mathrm{QL})$ of $117.93 \mathrm{nM}(\mathrm{S} / \mathrm{N}=10)$. Characterization of the sensor materials were done using field emission scanning electron microscopy (FE-SEM) and cyclic voltammetry (CV). Electrochemical investigations revealed that the proposed sensor displayed some significant benefits, including high effective surface area, numerous reactive sites and excellent electrochemical catalytic activity toward the oxidation and reduction of PRT. This sensor showed an excellent stability, selectivity, sensitivity, reproducibility and repeatability, recommending that the sensor was a promising one for the simultaneous determination of PRT and CN in real sample with acceptable recovery.

Keywords: Cetirizine; Paracetamol; Carbon paste electrode; Polymerization; Electroanalysis. 
(C) 2022 The Authors. Published by ESG (www.electrochemsci.org). This article is an open access article distributed under the terms and conditions of the Creative Commons Attribution license (http://creativecommons.org/licenses/by/4.0/). 